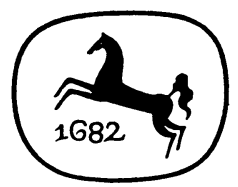

SAMMLUNG METZLER

REALIENBÜCHER FÜR GERMANISTEN

ABT.: LITERATURGESCHICHTE 


\title{
Bertolt Brecht
}

\author{
VON
}

REINHOLD GRIMM

MCMLXI

J.B. METZLERSCHE VERLAGSBUCHHANDLUNG 
ISBN 978-3-476-98848-5

ISBN 978-3-476-98847-8 (eBook)

DOI 10.1007/978-3-476-98847-8

(ㅇ) 1961 Springer-Verlag GmbH Deutschland

Ursprünglich erschienen bei J. B. Metzlersche Verlagsbuchhandlung und Carl Ernst Poeschel Verlag GmbH in Stuttgart 196r. 


\section{VORWORT}

Der vorliegende Band soll dem an Brecht Interessierten eine erste Einführung geben und dem Brecht-Forscher zur vorläufigen Orientierung dienen. Ich habe daher versucht, möglichst viel Daten- und Faktenmaterial zu bieten, auf eigene Deutung und Wertung dagegen zu verzichten. Die Literaturangaben verzeichnen die wichtigsten Veröffentlichungen über Brecht, soweit diese nicht in Nubels Bibliographie (vgl. S. XI), auf die jeweils verwiesen wird, aufgeführt sind. Sie berücksichtigen nach Möglichkeit auch noch Neuerscheinungen und Aufsätze des Jahres 1960. Zur Ergänzung ist außer Nubel (dessen Zusammenstellung selber keineswegs lückenlos ist) vor allem das Bertolt Brecht-Archiv in Berlin (vgl. S. 86) heranzuziehen, wo sich auch eine umfangreiche Sammlung von Rezensionen - über 75 Leitz-Ordner umfassend - und Photomaterial zu Leben und Werk Brechts befindet. Zahlreiche Einzelbesprechungen aus den letzten Berliner Jahren enthält die Zeitschrift 'Theater der Zeit‘. Ein Verzeichnis der Schallplatten mit Brecht-Aufnahmen gibt Willett (vgl. S. XII). Zu beachten ist schließlich, daß Hinweise am Schluß der einzelnen bibliographischen Abschnitte, die den Zusatz S. = Seite tragen, sich auf den vorliegenden Band beziehen. - Daß ich einen Großteil der Angaben im Brecht-Archiv nachprüfen konnte, verdanke ich einem Reisestipendium, das die Universität Erlangen mir in großzügiger Weise gewährte. Zu besonderem Dank bin ich Herrn Dr. HansJoachim Bunge verpflichtet, unter dessen Leitung das BrechtArchiv aufgebaut wurde: er unterzog sich freundlicherweise der Mühe, den biographischen Abriß im Manuskript zu lesen. Mein Dank gilt ferner den Herren Dr. F. X. Bayerl und Dr. H.Preiß sowie Fräulein Chr. Rubner, die das Register anfertigte. 


\section{INHALT}

LEBEN UND WERKE BREChTS I

I. Jugend (1898-1920) I

2. Die zwanziger Jahre (1920-1933) s

3. Im Exil (1933-1948) 27

4. Berlin (1948-1956) so

Probleme und Aufgaben der BRECHT-FORSCHUNG $\quad 68$

$\begin{array}{ll}\text { Register } & 89\end{array}$ 


\section{Abgekürzt zitierte Literatur}

\section{Werk-Ausgaben}

Versuche $=$ Bertolt Brecht Versuche, Heft I-I 5 . Davon sind erschienen: H. I-7 bei Kiepenheuer, Berlin 1930-1933; H. 9-1 5 in Parallelausgaben bei Suhrkamp, Berlin u. Frankfurt, und im Aufbauverlag, Berlin (Ost), I949-1956; H. I-8 wurden 1959 bei Suhrkamp (in 2 Bänden) neu gedruckt (H. 8, das 1933 nicht mehr erscheinen konnte, liegt nur in diesem Neudruck vor). Ein Sonderheft der "Versuche" erschien 1953 im Aufbauverlag.

Die "Versuche" bieten jeweils außer den Texten vielfach Materialien und Dokumente zur Werkgeschichte.

Ges. Wer Ke = Bertolt Brecht Gesa mmelte Wer ke. London, MalikVerlag 1938. (Nur zwei Bände sind erschienen.)

StüCKe = Bertolt Brecht StüCKe. I 2 Bde. Parallelausgaben bei Suhrkamp, bzw. im Aufbauverlag 1953-1959.

Die "Stücke" sind bei Suhrkamp in bis zu zwei Auflagen, im Aufbauverlag in bis zu vier Auflagen gedruckt worden. Innerhalb der Druckauflagen des Aufbauverlages zeigen die einzelnen Texte $z$. T. erhebliche Abweichungen voneinander.

Als Ergänzung zu den "Stücken " sind 6 Bde "Gedichte» angekündigt, von denen 3 Bde noch 1960 erscheinen sollen.

Schriften zum Theater = Bertolt Brecht Schriften zum TheaTER. Uber eine NICHT-ARIStotelische Dramatik, zusammengest. v. Siegfried Unseld. Suhrkamp I957 (= Bibliothek Suhrkamp 4I).

\section{Sammelbände, Sondernummern}

Adam AND EnCore $=A$ joint Brecht issue of the literary magazine ADAM and the theatre quarterly ENCORE. London, autumn 1956.

Articles et Études = Bertolt Brecht, i 898-1956: Articles et ÉtuDEs. Paris: Editeurs français réunis $1957 .^{* *}$

Bildband Weigel = Die Schauspiel erin Helene Weigel. Ein Fотовисн, hg. v.Wolfgang Pintzka. Berlin: Henschelverlag i 959.*

Brecht Friedensrat = Bertolt Brecht zum Gedenken. Hg. v. Deutschen Friedensrat, Berlin [1956].

Brecht Kulturbund = Bertolt Brecht. Hg. v. Deutschen Kulturbund, Berlin 1958.

Europe = Europe. Revue mensuelle. 35. Année, No 133/134, janvier/février 1957 [= Sonderheft Bertolt Brecht].* 
Quaderno = Quaderno del Piccolo Teatro della cittì di Milano. Mailand 1960 [ $=$ Sondernummer Bertolt Brecht].**

\SUF I bzw. $2<=$ SinN UND Form. BEITRÄGE ZUR Literatur: I. SONDERHEFT Bertolt Brecht, 1949; 2. SONDERHEFt Bertolt BRECHT, 1957.

Theaterarbeit $=$ Theaterarbeit. Sechs Aufführungen des Berliner Ensembles, hg. v. Berliner Ensemble (Leitung: Helene Weigel). Dresden: Dresdner Verlag i952.*

\section{Literatur über Brecbt}

Benjamin = Walter Benjamin Schriften, hg. v. Theodor W. Adorno u. Gretel Adorno unter Mitwirkung v. Friedrich Podszus. 2 Bde. Frankfurt: Suhrkamp 1955.

Bihalji-Merin = Oto Bihalji-Merin Bert Brecht. Pokušaj JedNOG SINTETIČNOG PORTRETA [ = Versuch eines synthetischen Porträts] in: KNJIžEVNOST (Belgrad) 20 (1955), $3 \mathrm{ff}$. [nun auch in O. B.-M. SusReti U MOgine vREMENom, Belgrad I $957,146 \mathrm{ff}$.].

Bloch = Ernst Bloch Das Prinzip HoffNung. 2 Bde. Frankfurt: Suhrkamp I959.

BRONNEN = ARNOLT BRONNEN GIBT ZU PROTOKOLL. BEITRÄGE ZUR GESCHICHTE DES MODERNEN SCHRIFTSTELLERS. hamburg: rowohlt I954.

Bunge $=$ Hans-Joachim Bunge ANTIGonemodell I948 von Bertolt Brecht und Caspar Neher. Zur Praxis und Theorie Des epischen (Dialektischen) Theaters Bertolt Brechts. Diss. Greifswald 1957 [Autorreferat in: /Wissenschaftliche Zeit-

* Enthält Bildmaterial zu Leben bzw. Werk Bertolt Brechts. Weiteres Bildmaterial u.a. in: S. d'Amico Storia DEL TEATRo Dramatico, Mailand ${ }^{4}$ i958, Bd IV; Die Schauspielerin Angelika Hurwicz. Ein Fotobuch, hg. v. W. Pintzka, Berlin 1960; Bertolt Brechts Dreigroschenbuch, Frankfurt i 960 ; Bertolt BRECHT UND DAS BERLINER Ensemble, hg. v. der Gesellschaft für kulturelle Verbindungen mit dem Ausland, Berlin 1958; Arnolt Bronnen TAge mit Bertolt Brecht. Die Geschichte einer UNVOLLENDETEN FreUNDSCHAFT, München 1960 ; T. Kulisiewicz ZEICHNUNGEN ZUR INSZENIERUNG DES BerLiner ENSEMbles: Der KaUkasische Kreidekreis, Berlin 1956; S. Melchinger Modernes Welttheater, Lichter und Reflexe, Bremen i 956; K. Pfützner Das revolutionäre Arbeitertheater in Deutschland igi 81933, in: ।Schriften zurTheaterwissenschaftı, hg. v. der Theaterhochschule Leipzig, Berlin I959, Bd I, 375 ff.; O. Schuberth Das BüHNenbild. Geschichte - Gestalt - Technik, München i955; außerdem in den Modellbüchern zu den einzelnen Stücken, soweit sie vorliegen, und in der Zeitschrift > Theater der Zeit .

** Konnte nicht eingesehen werden. 
schrift der Ernst-Moritz-Arndt-Universität Greifswald. Gesellschafts- und sprachwissenschaftliche Reihes 7 (1957/58), 236 ff.].

Chiarini $=$ Paolo Chiarini Bertolt Brecht. Bari: Editori Laterza I959 [ = Biblioteca di cultura moderna 538].

CrumbaCH $=$ Franz Hubert Crumbach Die Struktur des Epischen Theaters. Dramaturgie der Kontraste. Braunschweig: Waisenhaus-Buchdruckerei 1960 [ = Schriftenreihe der Pädagogischen Hochschule Braunschweig 8].

EssLin = Martin Esslin BRECHT - A CHOICE OF EVILS. A CRITICAL STUDY OF THE MAN, HIS WORK AND HIS OPINIONS. London: Eyre and Spottiswoode 1959 [veränderte amerikanische Fassung: Brecht. The Man And His Work. Garden City: Doubleday 1960].*

FassmanN $=$ Kurt Fassmann Brecht. Eine Bildbiographie. München: Kindler Verlag 1958.*

Fechter $=$ Paul Fechter Das europäische Drama. Geist und Kultur im Spiegel des Theaters. 3 Bde. Mannheim: Bibliogr. Institut $1956 \mathrm{ff} . *$

Fradkin = I. Fradkin Literatura nOvoj GERMANII, Moskau 1959. Gassner $=$ John Gassner The Theatre in our Times. A Survey OF the Men, Materials and Movements in the Modern Theatre. New York: Crown Publ. I954.

GEISSLER $=$ ZUR INTER PRETATION DES MODERNEN DRAMAS. BRECHTDürRENMATT-FrISCH, hg.v. Rolf Geißler u.a. Frankfurt: Diesterweg [1960].

Ghisselbrecht $=$ André Ghisselbrecht AINSI VA LE MONDE ET IL NE va pas bien, in: Europe [s. o.], 35. Année, No 133-34, janvierfévrier I957 [= Sondernummer BERTOLT BRECHr], $67 \mathrm{ff}$.

Gorelik = Mordecai Gorelik New Theatres for Old. New York: S. French ${ }^{8}$ I $949 .^{*}$

Grimm Struktur = Reinhold Grimm Bertolt Brecht. Die Struktur seines Werkes. Nürnberg: Verlag H. Carl ${ }^{2} 1960$.

Grimm Tragödie = Reinhold Grimm Ideologische Tragödie UNd TRAgödie der IdeOlogie. Versuch ÜBer eIN LeHrSTüCK voN Brecht, in: \Zeitschr. f. dt. Philol. 78 (I959), 394 ff.

HAAS $=$ Willy Haas BERT BRECHT. Berlin: Colloquium-Verlag 1958.

Hartung $=$ Günter Hartung ZUR episChen Oper Brechts UND WeILls, in: 'Wissenschaftliche Zeitschrift der Martin-LutherUniversität Halle-Wittenberg. Gesellschafts- und sprachwissenschaftliche Reihe< 8 (I 958/59), 659 ff.

Hilfsmaterial = Bertolt Brecht/Friedrich Wolf. HilfsMATERIAL FÜR DEN LITERATURUNTERRICHT AN DEN OBER- UND Fachschulen. Berlin: Verlag Volk und Wissen i955 [eine Neubearbeitung wird von H.-J. Bunge, W. Hecht und K. Rülicke vorbereitet].

Hill/Ley = Claude Hill and Ralph Ley The Drama of German Expressionism. A German english Bibliography. Chapel Hill: The University of North Carolina Press 1960. 
Hinck = Walter Hinck Die Dramaturgie des späten Brecht. Göttingen: Vandenhoeck \& Ruprecht ${ }^{2}{ }_{1} 960$ [ = Palaestra 229]; erweiterte und veränderte Fassung von H.s Diss. Problematik der Dramaturgie und Spielweise in Bert Brechts „epiSChEM Theater", Göttingen I956.

Hütt = Wolfgang Hütt Bertolt Brechts episches Theater und Probleme der bildenden Kunst, in: , Wissenschaftliche Zeitschrift der Martin-Luther-Universität Halle-Wittenberg. Gesellschafts- und sprachwissenschaftliche Reihes 7 (1957/58), 82 Iff.*

Ihering Theater = Herbert Ihering Bertolt Brecht Und Das Theater. Berlin: Rembrandt-Verlag 1959. *

IHERING JahrzehNTE $=$ Herbert Ihering Von ReINHARdt bIS Brecht. Vier Jahrzehnte Theater und Film. Berlin: Aufbauverlag $1958 \mathrm{ff}$. [bisher 2 Bände].

Ivernel $=$ Philippe Ivernel Pédagogie et politique chez BerTHOLD BRECHT, in: " Le théâtre moderne. Hommes et tendences, Etudes réunies et présentées par Jean Jacquot «, Paris: Centre national de la recherche scientifique i958, i75 ff.

Jens $=$ Walter Jens Statt einer Literaturgeschichte. Pfullingen: Neske ${ }^{3}$ I958 [ähnlich W. J. Protokoll über Brecht. Ein NeKROLOG, in: >Merkurs ro (1956), 943 ff.].

Kantorowicz $=$ Alfred Kantorowicz Deutsches Tagebuch. Erster Teil. München: Kindler Verlag 1959.

Kesting Brecht = Marianne [Wolf-]Kesting Bertolt Brecht in Selbstzeugnissen und Bilddokumenten. Hamburg: rowohlt I959.*

Kesting Episches Theater = Marianne [Wolf-]Kesting Das epIsche Theater. Zur Struktur des modernen Dramas. Stuttgart: E. Klett 1959; ursprünglich Diss. München I957.

KLOtZ = Volker Klotz Bertolt Brecht. Versuch üBER DAS Werk. Darmstadt: Gentner I957.

KöNigshof = Kaspar Königshof Über DEN Einfluss des EpISCHEN IN DER Dramatik, in: >Sinn und Form/ 7 (I955), $578 \mathrm{ff}$.

Kopetzki = Eduard Kopetzki Das Dramatische Werk Bertolt Brechts nach seiner Theorie vom epischen Theater. Diss. Wien I 949.

Kracauer = Siegfried Kracauer Von Caligari bis Hitler. Ein Beitrag zur Geschichte des deutschen Films. Hamburg: rowohlt I958.*

Leiser-AanderaA = Erwin Leiser und Johs. Aanderaa Bertolt Brecht. Oslo: i958.

Luthard $=$ Theodor Luthardt Vergleichende Studien zU Bertolt Brechts >Kleines Organon für das Theater<. VerSUCH EINER INTERPRETATION, ALS BEITRAG ZUR ZEITGENÖSSISCHEN Theatersituation gedacht. Diss. Jena i955.

Lüthy = Herbert Lüthy VOM ARMEN Bert BRecht, in: >Der Monats 4 (195I/52), H. 44, I Is ff.; auch in: >Preuvess 3 (1953), 
Nr 25, $30 \mathrm{ff}$. [französ.], in: >Tempo presenter I (I956), $495 \mathrm{ff}$. [ital.] u. ö.

MANN = Otto Mann B. B. - Mass oder Mythos? EIN Kritischer Beitrag über die Schaustücke Bertolt Brechts. Heidelberg: Rothe Verlag I958.

Melchinger = Siegfried Melchinger Theater Der Gegenwart. Frankfurt: Fischer ${ }^{3}$ I 960.

Müller = Joachim Müller Dramatisches und episches TheaTER. Zur ÄSTHETISCHEN ThEORIE UND ZUM BÜHNENWERK BERTOLT BRECHTS, in: >Wissenschaftliche Zeitschrift der FriedrichSchiller-Universität Jena. Gesellschafts- und sprachwissenschaftliche Reiher 8 (1958/59), $365 \mathrm{ff}$.

Nellhaus = Gerhard Nellhaus Bertolt Brecht. The Development of a Dialectical Poet-Dramatist. Diss. Harvard i 946. **

Niessen $=$ Carl Niessen Brecht auf Der Bühne. Köln 1959 [ = Ausstellungskatalog; demnächst in Buchform].*

Nubel = Walter Nubel Bertolt Brecht-Bibliographie, in: >SuF 2< [s. o.], $478 \mathrm{ff}$.

Piscator = Erwin Piscator Das politische Theater. Berlin: Schultz Verlag 1929.*

RINSER = Luise Rinser Der Schwerpunkt. Frankfurt: S. Fischer 1960.

RISMONDO = Piero Rismondo WER WAR BERT BREcht?, in: , Wort und Wahrheit< I I (1956), 85 s ff.

Rühle = Jürgen Rühle Das gefesselte Theater. Vom RevoLUTIONSTHEATER ZUM SOZIALISTISCHEN ReALISMUS. Köln: Kiepenheuer \& Witsch r957. *

Schaefer $=$ Heinz Schaefer Der Hegelianismus der Bert Brechtschen Verfremdungstechnik IN AbHängigkeit voN IHREN MAR XistisCHEN GRUNDlaGen. Diss. Stuttgart I 957.

Schöne $=$ Albrecht Schöne Bertolt Brecht. Theatertheorie UND DRAMatische Dichtung, in: >Euphorion 52 (I958), 272 ff.

SCHumaCher Versuche = Ernst Schumacher Die DRAMatischen Versuche Bertolt Brechts i9 18-1933. Berlin: Rütten \& Loening I955; ursprünglich Diss. Leipzig I953.

SchumaCher ER wird BLEIBEN = Ernst Schumacher ER wird BLEIBEN, in: >Neue dt. Lit.< 4 (1956), H. Io, I 8 ff.

Serreau = Geneviève Serreau Bertolt Brecht. Paris: Ed. L'Arche I955.*

Süsskind = W. E. Süßkind Bert Brecht. I 898-1956, in: Die großen Deutschen, hg. v. H. Heimpel, Th. Heuß u. B. Reifenberg, Bd V. Berlin: Propyläen-Verlag 1957, s io ff.

Thieme = Karl Thieme Des Teufels Gebetbuch? Eine AusEINANDERSETZUNG MIT DEM WERKE BERTOLT BRECHTS, in: >Hochland 29 (1932), $397 \mathrm{ff}$.

UhSE = Bodo UhSe VON ALTER UND NEUER WeISHeIt, in : >SuFく I I (1959), $420 \mathrm{ff}$. [nun auch in B. U. Gestalten und Probleme. Berlin: Aufbauverlag 1959]. 
Weber = Werner Weber Zeit ohne Zeit. Aufsätze zur LiteraTUR. Zürich: Manesseverlag I959.

Willett $=$ John Willett The Theatre of Bertolt Brecht. A Study from Eight Aspects. London: Methuen 1959. *

Wintzen Brecht $=$ René Wintzen Bertolt Brecht. Paris: Seghers I954.*

Wintzen Présence = René Wintzen Présence de Bertolt BRECHT, in: 'Documentse (Straßburg) 7 (I952), I I 3 ff.

Wirth = Andrzej Wirth Über die stereometrische Struktur DER BRechtschen Stücke, in: >SuF $2<$ [s. o.], $346 \mathrm{ff}$.

Znamenskaja = Galina Znamenskaja Bertolt Brecht. BiblioGRAFIČESKIJ UKASATEL'. Moskau I955.

Zwerenz = Gerhard Zwerenz Aristotelische und Brechtsche Dramatik. Versuch einer ästhetischen Wertung. Rudolstadt: Greifen-Verlag 1956. 